\title{
APUNTES PARA UNA TEORÍA CRÍTICA DE LAS RELACIONES DE GÉNERO EN EL CAPITALISMO
}

\section{NOTES FOR A CRITICAL THEORY OF GENDER RELATIONS IN CAPITALISM}

Facundo Nahuel Martín ${ }^{1}$

Fecha de recepción: 15 de mayo de 2017 - Fecha de aceptación: 17 de julio de 2017

\begin{abstract}
Resumen
Este trabajo se propone analizar las relaciones de género en el capitalismo desde la perspectiva de la teoría crítica de la sociedad. Articulando la lectura categorial de Marx de Moishe Postone y las contribuciones a la Wertkritik de Roswitha Scholz y los estudios de John D'Emilio, se sostendrá que el capitalismo guarda una relación contradictoria con el género. Por un lado, al hacer retroceder las relaciones de dominación personal, la sociedad capitalista genera condiciones para una pluralización históricamente novedosa de las relaciones de género. Por otro lado, con el capitalismo se produce una forma de patriarcado heterosexual que le es históricamente específica, ligada a la separación entre producción y reproducción. De esta doble situación se desprende la conclusión de que el capitalismo generó nuevas formas de opresión de género, pero también creó condiciones para su contestación crítica.

Palabras clave: Capitalismo, Patriarcado, Wertkritik, Género, Teoría Crítica.
\end{abstract}

\begin{abstract}
This paper intends to analyze the contradictory relations between capitalism and gender from the standpoint of critical social theory. Adressing Moishe Postone's categorial reading of Marx, Roswitha Scholz's contributions to Wertkritik and John D'Emilio's studies, it can be claimed that capitalism holds a contradictory relation towards gender. On one side, it undermines relations of personal domination, thus enabling conditionts for a pluralization of gender relations. On the other hand, capitalist society produces a historically specific form of heterosexual patriarchy, related to the separation between production and reproduction. Regarding gender relations, capitalism creates oppresive conditions and at the same time brings about new social possibilities. This leads to the conclusion that capitalism generated new forms of gender opression, but also laid the condictions for its critique.
\end{abstract}

Key Words: Capitalism, Patriarchy, Wertkritik, Gender, Critical Theory.

$1 \quad$ Universidad del Museo Social, Argentina, facunahuel@gmail.com 


\title{
Introducción a la problemática y estado de la cuestión
}

La teoría crítica de la sociedad se ha preocupado por analizar en forma vasta y comprehensiva a la modernidad capitalista, considerando aspectos como la formación social e histórica de la subjetividad, la psicología de las masas o las transformaciones en la familia. La atención a cuestiones como el antisemitismo, la relación entre la sociedad y la naturaleza o el vínculo de los individuos con la autoridad, permitieron a la teoría crítica configurar una ampliación, radicalización y en parte reformulación del pensamiento de inspiración marxista, buscando una mirada más integral y compleja de los movimientos contradictorios de la sociedad moderna. En Dialéctica de la Ilustración aparece una temprana consideración sobre la subordinación social y psíquica de las mujeres en la historia de la civilización occidental.

\begin{abstract}
El hombre debe salir a la vida hostil, debe actuar y luchar. La mujer no es sujeto. Ella no produce sino que cuida a los productores (...) La división del trabajo impuesta por el hombre le ha sido poco favorable: ha hecho de ella una personificación de la función biológica, una imagen de la naturaleza, la opresión de la cual ha sido el título de gloria de esta civilización (Adorno y Horkheimer, 2007, p. 267).
\end{abstract}

Esta temprana y lúcida consideración, sin embargo, no ha sido acompañada de un desarrollo teórico más sistemático y articulado sobre el vínculo entre la historia de la civilización moderna y la dominación masculina. ${ }^{2}$ Se hace necesario, precisamente en virtud de la amplitud de miras de la teoría crítica de la sociedad, profundizar esta problemática.

Por otra parte, durante los años 70 y 80 se dio un nutrido debate en torno a la relación entre capitalismo y dominación masculina. Una serie de feministas materialistas discutieron acerca del vínculo entre la opresión de las mujeres y la economía del plusvalor. ${ }^{3}$ Este debate, sin desaparecer, tendió a quedar relegado a partir de los años 90, de la mano de la más general "crisis del marxismo" y el retroceso general de la teoría crítica del capitalismo en los ámbitos tanto académicos como políticos. Al mismo tiempo aparecieron novedosos y potentes conceptos para la deconstrucción del género, como la teoría queer (Butler, 2006; 2007). Recientemente, sin embargo, ha existido una relativa reapertura del debate sobre capitalismo y relaciones de género. El trabajo de Federici (2004) sobre la acumulación originaria y la construcción de una dominación masculina específicamente moderna ha sido especialmente significativo en este contexto. Trabajos como los de Cinzia Arruzza $(2014 ; 2015)$ y sus discusiones con Oksala (2015), Farris (2015) y Manning (2015) dan cuenta también de esta renovada preocupación.

Finalmente, la propia teoría crítica del capitalismo ha experimentado importantes reformulaciones durante las últimas décadas. No sólo las teorías en torno al género se renovaron en los últimos decenios, sino que también la propia teoría crítica del capital ha producido importantes reformulaciones y actualizaciones teóricas. En particular, Moishe Postone propone una reformulación de la teoría crítica de la sociedad a partir de una original apelación al pensamiento de Marx. Esta lectura supone que la crítica de la economía política marxiana no se compone de conceptos económicos en sentido

2 Existe un número de interpretaciones feministas, particularmente de la obra de Adorno, que han intentado profundizar este planteo. Se destaca el volumen colectivo Feminist Interpretations of Theodor Adorno, editado por Renée Heberle (2006), así como Adorno, Culture and Feminsm editado por Maggie O’Neill (1999). Estos análisis enfatizan por lo general relaciones puntuales entre algunos conceptos de Adorno y algunas preocupaciones del feminismo contemporáneo. Sin embargo, no se detienen en la relación entre el capitalismo y la opresión de la mujer. Las excepciones a esto son los planteos de Roswitha Scholz (1999; 2005; 2014), de quien me ocuparé con mayor detalle en el trabajo.

3 Se puede mencionar a Christine Delphy (1985), Maria Rosa Dalla Costa y Selma james (1975), Shulamith Firestone (1976) como referentes de la renovación radical del feminismo en los años setenta. Para la relación entre capitalismo y género, puede verse a Lydia Sargent (1981), Iris Young (1992, 1997), Heidi Hartmann (1983) y Gayle Rubin (1986), Antoine Artous (1982). Para una introducción general a estos debates, puede verse Arruzza (2015). Recuperaré especialmente los aportes de Rubin y el debate entre Young y Hartmann, pero introduciré sus ideas directamente en la conclusión, cuando trate una evaluación crítica del pensamiento de Scholz. 
restringido, sino que forma una "broad and coherent social, political and cultural critique adequate to contemporary capitalist society [amplia y coherente crítica social, política y cultural adecuada a la sociedad capitalista contemporánea]" (Postone, 1993, p. 16). La propuesta de Postone, en continuidad con la teoría crítica de la sociedad de Adorno y Horkheimer, intenta dar cuenta globalmente de las formas de interacción social en la sociedad moderna. No se limita a una teoría económica ni a una lectura sobre la lucha de clases, sino que "express the forms of being [Daseinformen] and the determinations of existence [Existenzbestimmungen] of this specific society [expresa las formas de ser [Daseinformen] y las determinaciones de existencia [Existenzbestimmungen] de esta sociedad específica]" (Marx citado en Postone, 1993, p. 18). La teoría crítica, así concebida, "could be useful in analyzing the new social movements of the past two decades [podría ser útil para analizar los nuevos movimientos sociales de las últimas dos décadas]" (Postone, 1993, p. 37). Desde hace varias décadas, la política emancipatoria en sentido amplio guarda una relación más explícita y directa con una serie de experiencias de organización y lucha que no están centradas exclusivamente en el movimiento obrero, sino que politizan otros aspectos de la vida social. Estos movimientos se organizan en torno a cuestiones como el género, la identidad, la relación con el medio ambiente, la etnia o la cultura. Problemas como la opresión de género no pueden permanecer ajenos al análisis categorial de la sociedad capitalista. La lectura categorial, por su aspiración a proveer una lectura de la sociedad moderna que incluya factores objetivos y subjetivos, contemplando tanto las relaciones materiales como la subjetividad, debe tener especial interés (y recursos teóricos) para dar cuenta del vínculo entre opresión de género y capitalismo.

Por su parte, Roswitha Scholz, pensadora de la Wertkritik alemana, ha desarrollado una importante contribución a pensar las relaciones de género en el capitalismo, partiendo del planteo de Postone y de los trabajos de Robert Kurz, pero imprimiéndoles un importante giro adorniano (Scholz, 2006). Según esta autora, la crítica del valor como forma fetichizada de mediación social no está completa sin la crítica a la escisión del valor [Wertabspaltung] en la modernidad (2014). Se trata de una escisión de la vida social en dos grandes esferas: el trabajo productivo o creador de valor y las actividades reproductivas o de cuidados. El trabajo productivo es jerarquizado socialmente y asociado a la masculinidad, mientras que las actividades reproductivas son subalternizadas y vinculadas con lo femenino. De este modo, aparece una dominación particularista de los varones sobre las mujeres que es específica del capitalismo. La dominación por estructuras sociales anónimas se asocia con una forma de opresión de las mujeres que surge con las formas sociales de la modernidad del capital. El planteo de Scholz es sumamente importante porque es la primera formulación feminista desde una perspectiva afín a la lectura categorial. Sin embargo, sostendré que tiene algunas dificultades. Scholz no analiza cómo las relaciones sociales cuasi-objetivas propias del capitalismo separan efectivamente a los individuos de los lazos de dominación personal precapitalistas, resultando también posibilitadoras y habilitando instancias de pluralización de las relaciones de género.

A su vez, los estudios de D’Emilio (2006) sobre la relación entre el desarrollo del capitalismo y el surgimiento de la identidad gay en Estados Unidos, que recuperaré extensamente, permiten clarificar esta doble cara de la incidencia de las formas de mediación social capitalistas sobre las relaciones de género. Si el trabajo de Postone, por su nivel de abstracción, es indiferente al género; en cambio el trabajo de Scholz se concentra exclusivamente en las formas de dominación masculina propias de la modernidad capitalista, desconociendo cómo la propia dinámica del capital es posibilitadora de nuevas relaciones de género y nuevas luchas emancipatorias. Esto impide comprender el carácter dual (a la vez opresivo y posibilitador) de la relación entre capitalismo y género.

En este trabajo se intentará, sobre la base de un análisis de las formas de mediación social, que el capitalismo tiene una relación contradictoria con las relaciones de género. Esto significa que no es históricamente indiferente al género, pero tampoco guarda una correspondencia exclusiva con el tipo de dominación masculina y heterosexual que se plasmó en torno a la familia burguesa moderna. Por un lado, la sociedad capitalista ha generado históricamente un tipo específico de relaciones de género, que no son un mero resabio del pasado precapitalista y que se fundan en la familia patriarcal heterosexual 
como unidad normal de reproducción de la fuerza de trabajo. En torno a esta dominación se estructura una serie de privilegios masculinos y en el hogar y en el mundo del trabajo, así como la implantación de la familia nuclear heterosexual como modelo de relaciones de género. La familia burguesa como unidad de reproducción de la fuerza de trabajo se articuló históricamente como fundamento de un tipo de dominación masculina (una serie de privilegios adscriptos a los varones en relación con las mujeres) y de la exclusión social de las identidades sexo-genéricas irreductibles a la heterosexualidad. El fundamento de estas relaciones de género (y de dominación en torno al género) es la división capitalista entre la producción y la reproducción, división que no existe en otras sociedades. La familia presupuesta como unidad normal de reproducción de la fuerza de trabajo es el ámbito donde se anudan la opresión de la mujer (vinculada a las actividades reproductivas y subalternizada en el mundo del trabajo creador de valor) y la opresión de las identidades sexo-genéricas disidentes (incompatibles con la familia nuclear monógama y heterosexual como modelo). Analizaré las relaciones entre capitalismo y género desde el punto de vista de la constitución de un familiarismo capitalista, ligado a una forma de dominación patriarcal heterosexual que no es un mero resabio arcaico, sino que se constituye específicamente en la sociedad moderna.

Por otro lado (,) y contradictoriamente con lo anterior, la sociedad capitalista tiende a eliminar las relaciones de dominación personal o directa que caracterizan a las sociedades precapitalistas. Este cambio genera las condiciones para una pluralización históricamente inédita de las relaciones de género, para la experimentación, la visibilización y politización de identidades sexo-genéricas en disidencia con la normatividad de la familia heterosexual y patriarcal. En las relaciones sociales capitalistas las personas son dominadas por compulsiones sociales objetivas abstractas antes que por lazos de dependencia directa entre individuos o grupos. Articulando el planteo de Postone con los análisis históricos de John D’Emilio, se intentará explicar cómo los movimientos que cuestionan la dominación masculina y la heterosexualidad obligatoria son posibilitados contradictoriamente por la dinámica del capital y sus transformaciones en las relaciones sociales. Esto genera posibilidades novedosas para que constituyan identidades subjetivas y sexualidades de nuevas maneras, así como para poner en cuestión la dominación masculina heterosexual estructurada en torno a la división entre la producción y la reproducción.

El capitalismo, en síntesis, ha mantenido una relación estructuralmente contradictoria e históricamente cambiante con la sexualidad, el género y la familia. No puede considerárselo completamente ajeno al género, pero tampoco como exclusivamente un régimen de dominación masculina y heterosexual. La teoría crítica de la sociedad es, para Postone, una teoría sobre la contradicción entre las persistencias opresivas y las posibilidades liberadoras creadas por el capitalismo. Es posible formular una teoría crítica de las relaciones de género en el capitalismo, que conciba a la modernidad del capital como un fenómeno fundamentalmente contradictorio, esto es, que rompa con las visiones unilaterales que enfatizan ya su carácter de dominación, ya su carácter liberador.

\section{Capitalismo y pluralización del vínculo social}

Postone reinterpreta el pensamiento de Marx como una comprehensiva teoría crítica de la sociedad moderna en su conjunto. Según esta reinterpretación, con el surgimiento del capitalismo y las relaciones mediadas por el trabajo se dio una transformación de envergadura en la factura misma del ser social, cambiando cualitativamente la manera como están estructuradas las relaciones entre las personas. En las sociedades no organizadas a partir del intercambio universal de mercancías, las relaciones sociales son "abiertas". Esto significa que aparecen inmediatamente como tales, como relaciones entre personas o grupos de personas dados.

This is quite different from social formations in which commodity production and exchange do not predominate, where the social distribution of labor is effected by a wide variety of customs, traditional ties, overt relations of power, or, conceivablu, conscious decisions [Esto es bastante diferente de las formaciones sociales en las que la 
producción y el intercambio de mercancías no predominan, donde la distribución social del trabajo y sus productos es efectuada mediante una amplia variedad de costumbres, lazos tradicionales, relaciones abiertas de poder o, posiblemente, decisiones conscientes] (Postone, 1993, p. 149-150).

Si en las sociedades tradicionales la dominación se basa en relaciones de dependencia personal, en el capitalismo, en cambio, es la propia factura cuasi-objetiva y abstracta del nexo social fundado en el trabajo la que constituye la dominación. "Social domination in capitalism does not, on its most fundamental level, consist in the domination of people by other people, but in the domination of people by abstract social structures that people themselves constitute [La dominación social en el capitalismo no consiste, en este nivel fundamental, en la dominación de la gente por otra gente sino en la dominación de la gente por estructuras sociales abstractas que la misma gente constituye]" (Postone, 1993, p. 30). Las relaciones abiertas de las sociedades no capitalistas existentes hasta el momento implicaron muchas veces brutales formas de autoridad directa y dominación personal. En el capitalismo, esas formas "inmediatas" de dominación tienden a retroceder, pero para ser reemplazadas por una mediación anónima, abstracta y cuasi-objetiva, fundada en el trabajo y el valor. Bajo esa nueva forma de mediación, las personas pierden capacidad para controlar o modificar conscientemente sus vidas, su trabajo y su producción. La crítica del capitalismo, por lo tanto, no es una crítica de la propiedad privada y el mercado desde el punto de vista del trabajo sino una crítica del trabajo capitalista como fundamento del valor y, por ende, como categoría mediadora fundamental de la sociedad moderna constituida, que crea una lógica de dominación abstracta, impersonal y cuasi-objetiva.

A continuación, se intentará esbozar una interrogación sobre el vínculo entre las formas de mediación abstractas, cuasi-objetivas, características del capitalismo y las relaciones de género. En un primer movimiento argumentativo, siguiendo a John D’Emilio, trataré de mostrar que el capitalismo posibilita una pluralización relativa de las relaciones de género, en la medida en que la mediación social impersonal y abstracta da a los individuos algunas posibilidades para construir de manera contingente su trayectoria de vida personal. En un apartado subsiguiente, sin embargo, recuperaré el planteo de Roswitha Scholz para mostrar los límites de la pluralización de las formas de existencia en la sociedad capitalista y la gestación de una forma específicamente moderna de patriarcado. Con esto, se intentará ampliar la crítica de la dominación por la totalidad contradictoria para incluir, también, la dominación de las mujeres en la sociedad moderna.

D’Emilio estudia cómo, en Estados Unidos, el desarrollo del movimiento y de la identidad gay tuvo una relación no contingente con la difusión del trabajo asalariado. En la sociedad colonial de la Norteamérica precapitalista no había “espacio social” para un movimiento gay. Había, dice D’Emilio, prácticas homosexuales, que eran perseguidas (las fuentes jurídicas hablan de "sodomía" y "obscenidad"). Pero las personas no elaboraban esas prácticas como el fundamento de una identidad que pudiera politizarse y dar lugar a un movimiento social. La sociedad colonial se basaba fundamentalmente en una economía doméstica de subsistencia, donde las unidades familiares, de carácter patriarcal, no producían para el intercambio sino para el uso o consumo directos (el intercambio de mercancías, sin ser inexistente, tenía una menor importancia social). La dependencia personal con respecto a la autoridad patriarcal era un aspecto fundamental de la manera como estaba articulada la sociedad (la mediación social era de carácter abierto o directo). En un contexto donde las personas no podían ser social ni económicamente independientes de la familia patriarcal, algo como la "identidad” gay difícilmente podía desarrollarse, aunque hubiera, claro, prácticas homosexuales.

La evidencia de los registros de la corte y la iglesia en la Nueva Inglaterra colonial indica que el comportamiento homosexual masculino y femenino existió en el siglo diecisiete. El comportamiento homosexual, sin embargo, es diferente de la identidad homosexual. Simplemente no había un «espacio social» en el sistema de producción colonial que permitiera a los varones y a las mujeres ser gay. La supervivencia se estructuraba en torno a la participación en el núcleo familiar. Ciertamente había actos homosexuales -sodomía entre los varones, «obscenidad» entre mujeres- en 
los cuales las personas se involucraban, pero la familia era tan dominante que la sociedad colonial carecía incluso de la categoría de homosexual o lesbiana para describir a una persona (D`Emilio, 2006, p. 64).

Con la implantación del capitalismo, la situación cambia. Las familias dejan de ser unidades de producción, en la medida en que la economía de autosubsistencia es desplazada por el intercambio generalizado. Más gente puede entonces abandonar la familia en la que nació y ganarse la vida a través del trabajo asalariado. Ahora son las coacciones anónimas del mercado las que van a obligar a una mujer o un hombre a trabajar por un salario. Precisamente porque los constriñen mecanismos objetivos antes que autoridades personales, cada particular puede, en el marco de ciertas compulsiones objetivas dadas por la forma social, elegir una trayectoria de vida particular, contingente y diferente. Ahora se abre el "espacio social" para que las prácticas homosexuales den lugar lentamente a la formación de varias identidades, sobre la base de las cuales sería posible plantear demandas políticas y construir un nuevo movimiento social.

D’Emilio discute con la idea del "homosexual eterno". Las identidades sexuales, desde su punto de vista, son constituidas históricamente. Las identidades sexuales se forman históricamente en relación con procesos históricos amplios, ligados también al "carácter disruptivo del capitalismo en relación con la moral familiarista tradicional" (Ben, 2006, p. 54). La sociedad capitalista, por no estar basada en un nexo social de tipo personal o directo, habilita posibilidades nuevas para que las personas construyan trayectorias de vida particulares y diferentes. El desarrollo de los vínculos abstractos del capital sería entonces precondición de ciertas posibilidades de pluralización de las trayectorias de vida individuales y colectivas.

Al despojar al hogar de su independencia económica y fortalecer la separación entre sexualidad y procreación, el capitalismo creó las condiciones que permitían a algunos varones y mujeres organizar una vida personal en torno a su atracción erótico/emocional hacia personas del mismo sexo. El capitalismo ha hecho posible la formación de comunidades urbanas de lesbianas y varones gays, y más recientemente, la formación de una política basada en la identidad sexual (D’Emilio, 2006, p. 63-64).

A pesar de todo lo anterior, para D’Emilio "no podemos afirmar que se haya dado un golpe fatal al heterosexismo y la homofobia" (D’Emilio, 2006, p. 69). Las razones que da se relacionan con esta "resistencia al cambio" del heterosexismo obligatorio y la homofobia se relaciona con la "naturaleza contradictoria de la relación entre capitalismo y familia" (D’Emilio, 2006, p. 70). Si el capitalismo socava a la familia al liberar a los individuos de los lazos personales de dominación y fomentar el intercambio de mercancías como forma de mediación social, al mismo tiempo ha elevado a la familia a "fuente de amor, afecto y seguridad emocional" (D’Emilio, 2006, p. 70). Esta exaltación se relaciona con la relegación de las actividades reproductivas al ámbito familiar. A pesar de que esta separación es dinámica (ha habido una tendencia a la socialización creciente de la niñez y la crianza a manos de la escuela, los medios de comunicación, etc.), la separación entre la producción de valor (fuera del hogar) y la reproducción de la fuerza de trabajo (atribuida a la familia) ha permanecido como un pilar económico (y no sólo ideológico) de la sociedad burguesa. Una lectura que se concentre sólo en la disolución tendencial de los lazos de dominación personales y no analice cómo el capitalismo ha atribuido a la familia nuclear una responsabilidad central en la reproducción social, permanece insuficiente para comprender el nexo entre capitalismo, heterosexismo y dominación masculina.

Como dice Pablo Ben, "hoy puede plantearse que el texto de D’Emilio ha quedado presa de su época” (Ben, 2006, p. 54) en la medida en que no contempla más que la problemática lésbico/gay, excluyendo una mirada transexual/travesti/intersexual. Es un texto anterior a las importantes formulaciones de la teoría queer y las críticas a las políticas de la identidad en el propio movimiento LGBT. Estas limitaciones son importantes, pero no mellan el núcleo de su argumento. En efecto, la tesis de que la eclosión de los lazos de dominación personal contribuyó al cuestionamiento de la familia patriarcal heterosexual y posibilitó nuevas formas de la sexualidad, con las construcciones identitarias y las elaboraciones 
políticas correlativas, ofrece un marco poderoso para comprender cómo el capitalismo no sólo genera formas propias de opresión de género, sino que también hace posibles los planteos emancipatorios.

\section{Patriarcado productor de mercancías y escisión del valor}

En el apartado anterior se intentó desarrollar, partiendo de D’Emilio, la relación entre la aparición de las formas de mediación social capitalistas, basadas en compulsiones cuasi-objetivas antes que, en lazos de dependencia personales, y la emergencia de nuevas posibilidades para los particulares. Una vez que el nexo social no se funda en la dominación personal o directa, los particulares pueden construir sus identidades de maneras novedosas, posibilitadas en parte por la mutación de la forma social en la modernidad del capital. Estas posibilidades, empero, se ven constreñidas por los marcos de la totalidad social capitalista, esto es, vienen enmarcadas en el tipo de compulsiones anónimas y abstractas que caracterizan la dinámica de totalidad del capital.

En esta sección se recuperará la lectura feminista de Roswitha Scholz. La autora sostiene que el capitalismo acarrea una forma de dominación particularista de los varones sobre las mujeres. La igualdad formal de la época moderna viene asociada sin embargo a una implícita subordinación social de las mujeres, vinculada a las maneras diferentes como los géneros son construidos en torno a la creación de valor. Existe, por lo tanto, un patriarcado específicamente moderno o capitalista, ligado a las formas de mediación social en la modernidad.

Las formas de sociabilidad del capital y la igualdad formal entre las personas guardan una relación estructural. Sin embargo, la dominación patriarcal parece insistir decididamente en la sociedad moderna, a pesar de diversas conquistas del movimiento de mujeres. El patriarcado, ${ }^{4}$ aparentemente premoderno y precapitalista, parece persistir a pesar de la supuesta tendencia del capital a la igualdad formal, garantizada por la disolución de los lazos tradicionales de dependencia personal.

Scholz sostiene que el capitalismo acarrea una forma históricamente específica de patriarcado, al que podemos llamar "patriarcado productor de mercancías". Su punto de partida coincide con el de D’Emilio en torno a la mutación histórica de las formas de dominación en el capitalismo: "En las sociedades premodernas, por el contrario, se producía bajo otras relaciones de dominación (personales en vez de cosificadas por la forma de la mercancía) y principalmente para el uso" (Scholz, 2014, p. 48). En el capitalismo, en cambio, la dominación social se basa en la subordinación de las personas a la dinámica "tautológica" del capital que se autovaloriza, como "sujeto automático" basado en "mecanismos anónimos ciegos" (Scholz, 2014, p. 48) antes que en la violencia física directa ejercida por un grupo. La peculiaridad feminista de su análisis radica en que pasa de la crítica del valor a la crítica de la escisión del valor [Wert-Abspaltung]:

Con el valor o el trabajo abstracto no queda suficientemente especificada la forma fundamental del capitalismo en cuanto relación fetichista. También habría que dar cuenta del hecho de que en el capitalismo se producen actividades reproductivas que realizan sobre todo las mujeres. De acuerdo con esto, la escisión del valor remite a que las actividades reproductivas identificadas sustancialmente como femeninas, así como los sentimientos, los atributos y actitudes asociadas con ellas (emocionalidad, sensualidad, cuidado, etc.), están escindidos precisamente del valor/ trabajo abstracto (Scholz, 2014, p. 49).

La constitución de la lógica "abstracta", anónima y cuasi-objetiva de dominación en el capitalismo, que puede vincularse con la universal proclamación de la igualdad formal en la modernidad, sin embargo, se monta sobre una forma específicamente capitalista de dominación masculina. Esta

4 Gayle Rubin (1986, pp. 105 y ss.) ha cuestionado el uso de la categoría de patriarcado para dar cuenta de las relaciones de género en la modernidad. Según la autora, se trata de una categoría anacrónica que confunde todos los sistemas sexogénero histórico con una forma de dominación particular. Si bien su argumento puede ser correcto, mantendré el término porque es el más empleado por la bibliografía feminista y en particular por la propia Scholz. 
dominación se estructura a partir de la "escisión del valor", que masculiniza y jerarquiza el trabajo asalariado al tiempo que feminiza y desvaloriza las actividades reproductivas. La dominación patriarcal tiene una historia premoderna, pero "con la universalidad de la forma de la mercancía alcanzó una cualidad completamente nueva” (Scholz, 2014, p. 50). El capitalismo se construye sobre una división patriarcal de las actividades humanas, que asocia el trabajo creador de valor a la masculinidad (y a una serie de valores socialmente masculinizados, como la eficiencia, la competitividad, la agresividad); al tiempo que degrada y feminiza las actividades reproductivas, que aportan de manera indirecta a la reproducción de capital y se asocian a una serie de valores considerados femeninos, como el cuidado, la ternura, el afecto o incluso la irracionalidad.

Podría hablarse de manera algo exagerada del género masculino como del «género del capitalismo»; y, desde este trasfondo, cabría decir que una comprensión dualista de masculinidad y feminidad es la concepción dominante del género en la modernidad. El modelo civilizatorio productor de mercancías tiene su condición de posibilidad en la opresión de las mujeres (Scholz, 2014, p. 51).

La división del valor, principio estructurante de la sociedad capitalista, evidentemente no es estática, sino que asume una serie de formas históricas variables. En los actuales tiempos "posmodernos", por caso, las mujeres se ven sometidas a una "doble socialización": al tiempo que siguen siendo responsables de los cuidados y las actividades reproductivas en el hogar, participan también del trabajo asalariado fuera de casa. Esto, según Scholz, no cuestiona la raíz de las relaciones de género capitalistas, sino que las flexibiliza en un marco que no altera las bases formales de la WertAbspaltung [escisión del valor]. ${ }^{5}$

Según Scholz, la dominación social en el capitalismo es simultánea y co-originariamente dominación por la lógica del capital y dominación particularista de las mujeres por un nuevo tipo de patriarcado, asociado sistemáticamente a las formas modernas de reproducción social. Ambas dominaciones se articulan como lógicas diferentes, irreductibles la una a la otra, pero mediatizadas de manera sistemática. No es posible derivar la escisión del valor de la reproducción del valor, pero tampoco la inversa. Esto significa que la Wert-Abspaltung no es una nueva "contradicción fundamental" de la que se deriven dialécticamente las demás, pero tampoco es una mera "contradicción secundaria" derivada de una lógica de dominación abstracta más fundamental. La lógica capitalista que totaliza la sociedad en torno a la producción para el valor (fundada en la constitución de las relaciones sociales como cuasiobjetivas e independientes de los particulares) y la lógica de la escisión del valor (en virtud de la cual se desvaloriza el rol femenino) son irreductibles entre sí, pero se articulan sistemáticamente. "Dabei stehen der Wert und die Abspaltung in einem dialektischen Verhältnis zueinander. Es gibt keine Hierarchie der Ableitung [El valor y la escisión están en una relación dialéctica. No hay una jerarquía de derivación]” (Scholz, 2006, p. 2). Para comprender la imbricación entre la dominación particularista sobre las mujeres y la dominación abstracta plasmada en el valor y el trabajo, Scholz recurre a la crítica de Adorno a la "lógica de la identidad". Se trata de:

Kritik der Identitätslogik für sich in Anspruch, also die Kritik an einem deduktiven Denken, das von oben her Ordnung schaffen will und das Besondere, Kontingente, Differente, Nicht-Eindeutige einer Logik unterwerfen will [la crítica a un pensamiento deductivo, que quiere producir el orden desde arriba, y subordinar lo determinado, contingente, diferente, no-unívoco, a una lógica (Scholz, 2006, p. 5, cursivas originales).

$5 \quad$ Puede decirse, evidentemente, que el capitalismo apeló a diversas formas de trabajo asalariado femenino mucho antes de la actual "doble socialización" posmoderna. Esto no quita que exista una escisión del valor, ya que normalmente el trabajo de las mujeres ha sido minusvalorado (y peor pagado) en relación con el de los hombres. 
El capital, con su dinámica de totalidad, se imbrica con la escisión del valor, que sin embargo no se deduce orgánicamente de la lógica del capital. Ambas lógicas tienen una articulación compleja, con momentos contingentes e irreductibles entre sí.

Finalmente, el patriarcado capitalista se funda en una escisión entre la reproducción y la producción que se articula con la forma moderna de familiarismo. La naturalización de la familia como unidad de reproducción de la fuerza de trabajo, separada del trabajo productivo o creador de valor, está entrelazada con el patriarcado moderno. En la modernidad del capital, la familia nuclear heterosexual aparece como el ámbito de resguardo, contención afectiva y cuidados, delimitado de las lógicas competitivas, agresivas y el anonimato y la indiferencia de la esfera de la producción. Puede decirse, extrapolando el análisis de Scholz y para enfatizar la continuidad con la problemática del apartado anterior, que el capitalismo reforzó históricamente un tipo de dominación masculina ligado a la familia heterosexual, por lo que el patriarcado que le es específico es también heterosexista.

\section{Discusión. Capitalismo, familia y dominación masculina}

En los apartados anteriores se desarrollaron dos movimientos contradictorios. Por un lado, partiendo del análisis de D’Emilio, se sostuvo que el capitalismo, al construir un nexo social anónimo y cuasi-objetivo, socava a la familia patriarcal y posibilita la formación de identidades disidentes con la heterosexualidad obligatoria. Por otro lado, siguiendo a Scholz, se intentó mostrar que el capitalismo produjo una forma propia de dominación masculina fundada en la escisión del valor entre el trabajo y las actividades reproductivas. Esta situación dual plantea una contradicción en las relaciones entre capitalismo y género.

La discusión entre Heidi Hartmann e Iris Young constituye una referencia clásica en el debate sobre la relación entre capitalismo y patriarcado. Hartmann (1983) presenta lo que sería conocido como teoría del sistema dual: capitalismo y patriarcado serían dos sistemas de dominación relativamente autónomos entre sí, capaces experimentar relaciones de tensión pero que se han acomodado recíprocamente a lo largo del tiempo. "Sea o no la división patriarcal del trabajo, dentro y fuera de la familia, intolerable «en última instancia» para el capital, lo que sí es cierto es que está configurando al capitalismo hoy" (Hartmann, 1983, p. 22). Para Iris Young, en cambio, capitalismo y patriarcado no configuran dos sistemas sociales independientes sino un único "patriarcado capitalista".

Una de las características que definen al capitalismo es la separación de la actividad productiva de las relaciones de parentesco con la consiguiente creación de dos esferas de vida social. Este planteamiento y el mostrar cómo esta separación ha creado una situación histórica única para la mujer, ha sido uno de los logros principales del análisis feminista socialista (Young, 1992, p. 4).

En la sociedad capitalista, como se intentó reconstruir a partir del planteo de Scholz más arriba, las actividades reproductivas se escinden de las productivas (en términos de producción de valor), separando la economía de las relaciones de parentesco y constituyendo la familia nuclear burguesa como unidad de la reproducción social. Esta tesis no implica que se pueda derivar lógicamente, partiendo de la forma mercancía o la ley del valor, la dominación masculina. La razón por la que es necesaria una teoría unitaria es que no puede definirse la dominación patriarcal que caracteriza a la sociedad moderna en términos autónomos con respecto a las categorías de la sociedad capitalista. Las formas de opresión de género específicas de la modernidad están imbricadas con las categorías sociales capitalistas, en particular con la separación entre la producción y la reproducción.

El capitalismo tiene efectos contradictorios sobre el género. No hay dos sistemas autónomos, pero el capitalismo tampoco ha operado unilateral y exclusivamente como refuerzo de la familia nuclear y la dominación masculina. La dominación masculina y el heterosexismo obligatorio propios de la sociedad moderna se anudan con la división moderna entre producción (trabajo creador de valor) y reproducción. El capitalismo escinde estas dos esferas de la actividad humana, vinculando de modo 
prioritario (aunque también flexible) a la primera con la subjetividad masculina y la segunda con la femenina. Esta escisión, si bien es históricamente variable, explica también que la inclusión de las mujeres en el mercado de trabajo se realiza bajo condiciones de subordinación, tanto en los niveles salariales como en el acceso a posiciones de responsabilidad. Las identidades disidentes con la familia heterosexual, por su parte, también tendieron a ser excluidas o subalternizadas con la división capitalista entre producción y reproducción.

Antoine Artous ha precisado la naturaleza de este vínculo contradictorio desde el punto de vista de la situación de la mujer. "Hay que guardarse de tener una visión lineal, unilateral, en cuanto al proceso aportado por la sociedad capitalista respecto de la vieja sociedad" (Artous, 1982, p. 47). La difusión de las relaciones sociales objetivadas y basadas en la igualdad formal, propia del capitalismo, efectivamente incide en las relaciones de género y hace posibles luchas igualitaristas. La mujer recluida en la esfera de la reproducción o aceptada en forma subordinada en el mundo del trabajo, sin embargo, es reconocida como un individuo jurídicamente igual. Esto genera las condiciones para el cuestionamiento de los privilegios particularistas masculinos ligados históricamente al surgimiento del capitalismo y articulados con él. Si el capitalismo genera relaciones de dominación masculina heterosexual a partir de la familia como unidad de reproducción, también posibilita la crisis de esa familia. Dado que el capital es históricamente flexible con respecto a las formas concretas de reproducción de la fuerza de trabajo, puede crear el espacio social para el cuestionamiento del familiarismo instituido y reforzado por el propio capitalismo. "Nada nos permite afirmar que la familia en sí, como lugar de reproducción de la fuerza de trabajo, sea indispensable para el funcionamiento del sistema" (Artous, 1982, p. 82). Esta posibilidad, sin embargo, no se ha realizado en la actualidad y "el trabajo doméstico es todavía una necesidad importante para la reproducción" lo que se manifiesta en la "doble jornada que soporta la mujer trabajadora" (Artous, 1982, p. 85). Artous sostiene que el despliegue del capitalismo se asoció históricamente a una forma de patriarcado (y de dominación heterosexual, podemos agregar) históricamente determinada, pero también generó las condiciones para su impugnación. La constitución del familiarismo capitalista, ligado a formas de dominación masculina y heterosexual específicas, pues, coexiste con un proceso contrario que posibilita la pluralización de las relaciones de género y la contestación de la dominación masculina.

\section{Conclusión. Reformular la crítica inmanente}

Este trabajo recorrió dos grandes movimientos conceptuales. Primero puso en relación la relectura categorial postoniana con el estudio de D’Emilio sobre la historia del movimiento gay. Se sostuvo que sólo con la constitución de las formas de mediación social impersonales, anónimas y cuasi-objetivas características de la modernidad del capital, pudieron construirse movimientos sociales fundados en la reivindicación de una identidad gay. Esto se debe a que, para que las personas alcanzaran a elaborar sus deseos homosexuales en términos de una identidad diferenciada, fue una precondición la destitución de los lazos de dominación personal y su reemplazo por vínculos anónimos y abstractos. La mutación capitalista de las formas del nexo social habilita, así, potencialidades para la construcción de nuevas formas de subjetividad y, por ende, de identidades sexo-genéricas novedosas.

En el segundo gran movimiento conceptual se recuperaron los planteos Roswitha Scholz, analizando el vínculo entre la impostación del valor como lógica social y la escisión del valor en la modernidad, que masculiniza el trabajo productivo y feminiza las actividades domésticas, degradándolas como inferiores. Se trató entonces de reconstruir cómo la lógica de la totalidad del capital se asocia a formas particulares de dominación, que no son un mero derivado lógico pero que han estado históricamente ligadas a ella.

Finalmente, se sostuvo que la división entre producción y reproducción (y la adscripción de esta última a la familia y la mujer) es el ámbito donde la opresión de género se anuda con el capitalismo. Al separar estas dos esferas, la sociedad capitalista construyó la familia heterosexual nuclear monógama 
como unidad normal de reproducción de la fuerza de trabajo. Así se estructura un tipo de dominación patriarcal específica del capitalismo, que explica el rol subordinado de las mujeres en el mundo del trabajo, así como su adscripción a la esfera de la reproducción. En torno a este familiarismo capitalista se estructura también la subalternización de las identidades genéricas disidentes con la heterosexualidad obligatoria (presuponer la familia nuclear como unidad normal de reproducción es instituir la heterosexualidad como norma social).

Comprender el proceso dual del capitalismo con respecto al género ilumina algunas cuestiones importantes sobre el sentido de la teoría crítica de la sociedad. Primero, es falso que el capitalismo sea históricamente indiferente al género. La crítica del capitalismo no puede limitarse a cuestionar la dominación de las personas por estructuras sociales fetichizadas, objetivadas y que se reproducen con prescindencia de los individuos. Es preciso también cuestionar las formas de dominación particularista y los privilegios sectoriales que se asocian históricamente a la expansión del capital y se configuran en torno a sus categorías sociales. En particular, la opresión de las mujeres y las identidades no heterosexuales, aparece como un correlato duradero de la separación capitalista entre producción y reproducción.

Segundo, esta lectura permite delinear los contornos de una teoría crítica de las relaciones de género. La sociedad capitalista ha gestado tanto una forma propia de dominación heterosexual masculina, como las condiciones de posibilidad para cuestionar esa dominación. La teoría crítica, en este contexto de reformulación parcial, apunta por lo tanto en una doble dirección. Por un lado, aspira a superar las coacciones abstractas y objetivadas que estructuran al capitalismo en sí mismo. Por el otro, se dirige contra las formas de dominación particularista que se han asociado históricamente a la dominación del capital y que componen con éste un sistema flexible y mutable. No se trata sólo de discutir cómo las personas son oprimidas por un sistema de coacciones fetichizado, sino también de poner en cuestión los privilegios de grupo que ese sistema de coacciones reforzó y reformuló históricamente.

\section{Referencias}

Adorno, Theodor y Horkheimer, Max (2007). Dialéctica de la Ilustración. Madrid: Akal.

Arruzza, Cinzia (2014). "Remarks on Gender". En, Viewpoint Magazine, 2014. Recuperado en: https:// viewpointmag.com/2014/09/02/remarks-on-gender/

Arruzza, Cinzia (2015). Las sin parte. Matrimonios y divorcios entre feminismo y marxismo. Madrid: Syllone.

Artous, Antoine (1982). Los orígenes de la opresión de la mujer. Barcelona: Editorial Fontamara.

Dalla Costa, María Rosa y James, Selma (1975). El poder de la mujer y la subversión de la comunidad. Madrid: Siglo XXI.

Delphy, Christine (1985). Por un feminismo materialista. El enemigo principal y otros textos. Barcelona: La Sal.

Firestone, Shulamith (1976). La dialéctica del sexo. Barcelona: Kairós.

Farris, Silvia (2015). "The Intersectional Conundrum and the Nation-State". En, Viewpoint Magazine. Recuperado en: https://viewpointmag.com/2015/05/04/ the-intersectional-conundrum-and-the-nation-state/

Federici, Silvia (2004). Calibán y la bruja. Mujeres, cuerpo y acumulación originaria. Madrid: Traficantes de Sueños.

Hartmann, Heidi (1983). "Un matrimonio mal avenido: hacia una unión más progresiva entre marxismo y feminismo". En, Fundación Rafael Campanas (publicación virtual). Recuperado en: http:// www.fcampalans.cat/uploads/publicacions/pdf/88.pdf

Heberle, Reneé (2006) (ed.). Feminist Interpretations of Theodor Adorno. Pennsylvania: Pennsylvania State University Press. 
Manning, FTC (2016). "Closing the Conceptual Gap: a Response to Cinzia Arruzza's «Remarks on Gender»”. En, Viewpoint Magazine. Recuperado en: https://viewpointmag.com/2015/05/04/ closing-the-conceptual-gap-a-response-to-cinzia-arruzzas-remarks-on-gender/

Oksala, Johanna (2016). "Capitalism and Gender Opression: Remarks on Cinzia Arruza's «Remarks on Gender»”. En, Viewpoint Magazine. Recuperado en: https://viewpointmag.com/2015/05/04/ capitalism-and-gender-oppression-remarks-on-cinzia-arruzzas-remarks-on-gender/

O’Neill, Maggie (1999). Adorno, Culture and Feminsm. Nueva York: Sage.

Postone, Moishe (1993). Time, Labor and Social Domination. Cambridge: Cambridge University Press.

Rubin, Gayle (1986). "El tráfico de mujeres: notas sobre la «economía política» del sexo". En, Revista nueva antropología, Año VII, Nro 30, UNAM, pp. 95-145.

Sargent, Lydia (ed.) (1981) Women and Revolution: a Discussion of the Unhappy Marriage of Marxism and Feminism. Black Rose Books: Boston.

Scholz, Roswitha (1999) "Wert und Geschlechterverhältnis", publicación virtual. Recuperada en: http:// www.exit-online.org/textanz1.php?tabelle=autoren\&index=21\&posnr=37\&backtext1=text1.php

Scholz, Roswitha (2006). "Die Theorie des Geschlechtichen Abspaltungs und die Kritische Theorie Adornos" publicación virtual. Recuperada en: http://www.exit-online.org/textanz1.php?tabelle=a utoren\&index=25\&posnr=189\&backtext1=text1.php

Scholz, Roswitha (2014). "El patriarcado productor de mercancías. Tesis sobre capitalismo y relaciones de género". En Constelaciones. Revista de Teoría Crítica, N³, pp. 44-60. Recuperada en: http:// www.constelaciones-rtc.net/05/05_04.pdf

Young, Iris (1992). "Marxismo y feminismo, más allá del matrimonio infeliz (una crítica al sistema dual)". En, El cielo por asalto, Año II, No4, pp. 43-69. 